\title{
A Novel Analysis of the Smooth Curve with Constant Width Based on a Time Delay System
}

\author{
Teng Fu and Yusheng Zhou * \\ School of Mathematics and Statistics, Guizhou University, Guiyang 550025, China; gs.tfu20@gzu.edu.cn \\ * Correspondence: yszhou@gzu.edu.cn
}

check for

updates

Citation: Fu, T.; Zhou, Y. A Novel Analysis of the Smooth Curve with Constant Width Based on a Time Delay System. Mathematics 2021, 9, 1131. https://doi.org/10.3390/ math9101131

Academic Editor: Josef Mikeš

Received: 8 April 2021

Accepted: 14 May 2021

Published: 17 May 2021

Publisher's Note: MDPI stays neutral with regard to jurisdictional claims in published maps and institutional affiliations.

Copyright: (c) 2021 by the authors. Licensee MDPI, Basel, Switzerland. This article is an open access article distributed under the terms and conditions of the Creative Commons Attribution (CC BY) license (https:// creativecommons.org/licenses/by/ $4.0 /)$.

\begin{abstract}
In this paper, we analyze the $C^{\infty}$ smooth curve of constant width using the characteristic equation of a time delay system. We prove that a closed convex curve must be a circle if it is still a smooth curve of constant width after taking any number of derivatives. Finally, the simulation results are presented for analyzing the influence of derivative orders on a smooth non-circular curve of constant width.
\end{abstract}

Keywords: smooth curve of constant width; characteristic equation; time delay system; $C^{\infty}$ smooth

\section{Introduction}

The curves of constant width are a type of interesting curve that can be used to design precise mechanical structures [1]. Since Euler first proposed the concept of the curve of constant width, researches of the non-circular curve of constant width have attracted extensive attention [1-4]. In 1876, an engineer named Reuleaux constructed a curve of constant width that was based on an equilateral triangle, which is now known as the Reuleaux triangle. In 1997, Rabinowitz [5] investigated the non-circular curve of constant width and found its parametric equation, so as to plot the curve with a graphing calculator. In 2010, Paciotti [6] studied the curves of constant width and the shadows that they cast, and then used the shadows to distinguish different curves. In 2011, Xu et al. [7] used an isosceles trapezoid to construct another curve of constant width. This basic construction process can be generalized to construct curves of constant width that are based on special $2 n$-sided polygons with $\mathrm{N}$ groups parallel to the opposite sides. In 2015, Yang [8] constructed a non-circular curve of constant width using the opposite top sector, and the obtained curve of constant width included the results by Reuleaux and [7]. Additionally, Mozgawa [9] provided a generalization of the theorem characterizing ovals of constant width proved by Mellish [10]. Moreover, Resnikoff [11] investigated the curves and surfaces of constant width by means of Fourier series, with some additional results regarding general ovals. However, the main drawback of these methods is that the constructed curves of constant width are piecewise smooth. To address this issue, on the basis of the piecewise smooth curve, a $C^{1}$ curve of constant width with better smoothness can be constructed using the outer parallel lines [12]. In order to obtain a smoother curve of constant width, a class of $C^{\infty}$ smooth non-circular curves of constant width is constructed using the tangent polar coordinates of the plane curve based on the Minkowski support function [13]. However, in terms of these constructions, the first derivative of the parametric equation yields a curve with a self-intersection point, instead of a $C^{\infty}$ smooth curve.

Being motivated by the above analysis, it is natural to propose the following problem: whether there exists a $C^{\infty}$ smooth non-circular curve of constant width, such that its parametric equation after taking any number of derivatives still represents a smooth curve of constant width. In this paper, we aim to prove that there is no $C^{\infty}$ smooth non-circular curve of constant width in theory, so that the parametric equation after taking any number of derivatives is still a smooth curve of constant width. Moreover, the simulation results are proposed to further illustrate this hypothesis. 
The remainder of this paper is structured as follows. In Section 2, the related preliminaries and a hypothesis are presented. Section 3 provides the proof of the proposed hypothesis. Simulation results are reported and discussed in Section 4. Finally, some conclusions are given in Section 5.

\section{Preliminaries and a Hypothesis}

Let $\Gamma$ be a smooth closed curve in the plane $R^{2}$, and define an arbitrary point in the region that is bounded by the curve $\Gamma$ as the origin point of the plane $R^{2}$. A positive oriented coordinate system is established as XOY. $\theta$ represents the angle between the positive direction of the $O X$ axis and the perpendicular line that corresponds to the tangent line of $\Gamma$. Moreover, $P(\theta)$ denotes the distance from the origin $O$ to the tangent line of $\Gamma$, which is called the support function of the curve $\Gamma$.

Setting $(x, y)$ as a point on $\Gamma$, it follows from the support function $P(\theta)$ that

$$
x \cos \theta+y \sin \theta-P(\theta)=0 .
$$

Differentiating both sides of (1) with respect to $\theta$ gives

$$
-x \sin \theta+y \cos \theta-\dot{P}(\theta)=0 \text {. }
$$

When combining (1) with (2), the parametric equation of $\Gamma$ can be formulated by

$$
\left\{\begin{array}{l}
x=P(\theta) \cos \theta-\dot{P}(\theta) \sin \theta, \\
y=P(\theta) \sin \theta+\dot{P}(\theta) \cos \theta,
\end{array} \quad \theta \in[0,2 \pi) .\right.
$$

If $\Gamma$ is a smooth closed convex curve, then its support function should satisfy the following necessary and sufficient conditions.

Lemma 1 ([14]). The function $P(\theta)$ of period $2 \pi$ is the support function of a smooth closed convex curve, if and only if

$$
P(\theta)+\ddot{P}(\theta)>0, \quad \theta \in[0,2 \pi) .
$$

Now, we would like to consider an interesting curve: a smooth curve of constant width. First, we present the definition of the width of a simple closed convex curve.

Definition 1. The width function of a closed convex curve $\Gamma$ is defined as

$$
D(\theta)=P(\theta)+P(\theta+\pi) .
$$

If $D(\theta)=d$ is a constant, then $\Gamma$ is called a curve of constant width.

The $C^{\infty}$ smooth curve of constant width is the research objective of this paper. The so-called $C^{\infty}$ smooth curve means that each component of its parametric equation is infinitely differentiable, namely, $P(\theta)$ is infinitely differentiable. At present, many researchers have proposed several methods to construct curves of constant width. However, these constructed curves of constant width either have corners, such as the Reuleaux polygon, or have better smoothness derived from outer parallel lines, but the derivative of which is not smooth again. In addition, there are also some curves of constant width that can be infinitely differentiated, whereas, after several derivatives the resulting equations no longer represent curves of constant width.

Accordingly, it is natural to propose the following hypothesis.

Hypothesis 1. For a $C^{\infty}$ smooth curve $\Gamma$ of constant width, if its parametric equation is still a smooth curve of constant width after any number of derivatives, then the curve $\Gamma$ must be a circle. 


\section{The Proof of the Hypothesis}

Assume that the width of the $C^{\infty}$ smooth curve $\Gamma$ is $d$, namely,

$$
P(\theta)+P(\theta+\pi)=d,
$$

which is equivalent to

$$
P(\theta)+P(\theta-\pi)=d .
$$

After differentiating both sides of (7) with respect to $\theta$, one has

$$
\dot{P}(\theta)+\dot{P}(\theta-\pi)=0,
$$

which stands for a neutral delay differential equation.

Setting $P(\theta)=\tilde{C} \mathrm{e}^{\lambda \theta}$, and then substituting it into (8) gives the following characteristic equation

$$
\lambda \mathrm{e}^{\lambda \theta}+\lambda \mathrm{e}^{\lambda(\theta-\pi)}=0,
$$

which yields

$$
1+\mathrm{e}^{-\lambda \pi}=0 \quad \text { or } \quad \lambda=0 .
$$

When $\lambda=0$, we obtain $P(\theta)=\tilde{C}=\frac{d}{2}$. In this case, $\Gamma$ is a circle of radius $\frac{d}{2}$. When $\lambda \neq 0$, it is obvious that $\lambda$ cannot be a real number. Accordingly, when setting $\lambda=\alpha+\omega i$ and substituting it into (10), one has

$$
\mathrm{e}^{-\pi \alpha}[\cos (\omega \pi)-i \sin (\omega \pi)]=-1 .
$$

It follows from (11) that

$$
\left\{\begin{array}{l}
\mathrm{e}^{-\pi \alpha} \cos (\omega \pi)=-1 \\
\mathrm{e}^{-\pi \alpha} \sin (\omega \pi)=0
\end{array}\right.
$$

which implies

$$
\left\{\begin{array}{l}
\sin (\omega \pi)=0, \\
\cos (\omega \pi)=-1, \quad \mathrm{e}^{-\pi \alpha}=1 .
\end{array}\right.
$$

Furthermore, we obtain

$$
\alpha=0, \quad \omega=2 k+1, \quad k \in Z .
$$

Therefore, the general solution of (8) is expressed as

$$
P(\theta)=C_{1} \cos (\omega \theta)+C_{2} \sin (\omega \theta)+C_{3} .
$$

When substituting (15) into (6), one has

$$
C_{3}=\frac{d}{2} .
$$

It follows from (15) and (16) that

$$
P(\theta)=C \sin (\omega \theta+\varphi)+\frac{d}{2},
$$


where $C=\sqrt{C_{1}^{2}+C_{2}^{2}}$ with constants $C_{1}, C_{2}$ being decided by the initial condition, $\varphi$ is an angle, and its value can be derived from $\sin \varphi=\frac{C_{1}}{\sqrt{C_{1}^{2}+C_{2}^{2}}}$ or $\cos \varphi=\frac{C_{2}}{\sqrt{C_{1}^{2}+C_{2}^{2}}}$. By using Lemma 1, we see that the smooth curve $\Gamma$ is a closed convex curve, if and only if

$$
\frac{d}{2}-C\left(\omega^{2}-1\right)>0
$$

For simplicity, we assume that the support function is

$$
P(\theta)=C \sin (\omega \theta)+\frac{d}{2} .
$$

Substituting (19) into (3) gives

$$
\left\{\begin{array}{l}
x=\left[\frac{d}{2}+C \sin (\omega \theta)\right] \cos \theta-C \omega \cos (\omega \theta) \sin \theta, \\
y=\left[\frac{d}{2}+C \sin (\omega \theta)\right] \sin \theta+C \omega \cos (\omega \theta) \cos \theta .
\end{array}\right.
$$

Equation (20) can be rewritten as

$$
\left\{\begin{array}{l}
x=\frac{d}{2} \cos \theta+\frac{C}{2}[\sin [(\omega+1) \theta]+\sin [(\omega-1) \theta]]-\frac{C \omega}{2}[\sin [(\omega+1) \theta]-\sin [(\omega-1) \theta]] \\
y=\frac{d}{2} \sin \theta+\frac{C}{2}[\cos [(\omega-1) \theta]-\cos [(\omega+1) \theta]]+\frac{C \omega}{2}[\cos [(\omega+1) \theta]+\cos [(\omega-1) \theta]]
\end{array}\right.
$$

In what follows, we would like to discuss the curves $\Gamma$ described by (21) in different values of $\omega$. From (14), we see that $\omega=2 k+1, k \in Z$. Therefore, we will analyze the two cases of $\omega=1$ and $\omega>1$, respectively. When $\omega=1$, (21) becomes

$$
\left\{\begin{array}{l}
x=\frac{d}{2} \cos \theta \\
y=\frac{d}{2} \sin \theta+C,
\end{array}\right.
$$

which implies that the curve $\Gamma$ is a circle.

When $\omega>1$, taking the $n$-th derivative of (21) with respect to $\theta$ gives

$$
\left\{\begin{aligned}
x^{(n)}= & \frac{d}{2} \cos \left(\theta+\frac{1}{2} n \pi\right)+\frac{C}{2}\left[\sin \left[(\omega+1) \theta+\frac{1}{2} n \pi\right](\omega+1)^{n}+\sin \left[(\omega-1) \theta+\frac{1}{2} n \pi\right](\omega-1)^{n}\right] \\
& -\frac{C \omega}{2}\left[\sin \left[(\omega+1) \theta+\frac{1}{2} n \pi\right](\omega+1)^{n}-\sin \left[(\omega-1) \theta+\frac{1}{2} n \pi\right](\omega-1)^{n}\right], \\
y^{(n)}= & \frac{d}{2} \sin \left(\theta+\frac{1}{2} n \pi\right)+\frac{C}{2}\left[\cos \left[(\omega-1) \theta+\frac{1}{2} n \pi\right](\omega-1)^{n}-\cos \left[(\omega+1) \theta+\frac{1}{2} n \pi\right](\omega+1)^{n}\right] \\
& +\frac{C \omega}{2}\left[\cos \left[(\omega+1) \theta+\frac{1}{2} n \pi\right](\omega+1)^{n}+\cos \left[(\omega-1) \theta+\frac{1}{2} n \pi\right](\omega-1)^{n}\right] .
\end{aligned}\right.
$$

From (23), we see that, when $n$ is large enough, the term containing $(\omega+1)^{n}$ plays a predominant role in the parametric equation. In this case, (23) can be approximated to the following equation

$$
\left\{\begin{array}{l}
x^{(n)}=\frac{C}{2}(1-\omega)(\omega+1)^{n} \sin \left[(\omega+1) \theta+\frac{1}{2} n \pi\right], \\
y^{(n)}=\frac{C}{2}(\omega-1)(\omega+1)^{n} \cos \left[(\omega+1) \theta+\frac{1}{2} n \pi\right],
\end{array} \quad \theta \in[0,2 \pi) .\right.
$$

System (24) comprises a circle of radius $\left|\frac{C}{2}(1-\omega)(\omega+1)^{n}\right|$, and this circle goes around $(\omega+1)$ times when $\theta \in[0,2 \pi)$. As a result, when $n$ is large enough, the trajectory of system (24) is no longer a simple closed curve.

From the above analysis, we see that $\Gamma$ is still a $C^{\infty}$ smooth curve of constant width after taking any times of derivatives, if and only if $\omega=1$. This proves the hypothesis.

Remark 1. Although different non-circular smooth curves of constant width have been constructed in the literature, the obtained curves after taking any number of derivatives have not been further studied. From the proof procedure shown above, it can be seen that a non-circular curve of constant width is no longer a simple closed curve after taking enough derivatives, although it can be taking 
any number of derivatives. However, even though it does not represent a simple closed curve after taking many times of derivatives, the resulting curve, after taking enough derivatives, can be approximated to a circle that has turned $(\omega+1)$ times.

\section{Simulation Results}

In this section, we will present some simulation results to illustrate the main result of this paper.

First, for the support function (19), we set $C=1, d=100, \omega=3,5,7,9$. When $\omega=3,5,7, \Gamma$ in (20) represents a smooth curve of constant width, respectively, as can be seen in Figure 1a. However, for $\omega=9$, since (18) is not satisfied, $\Gamma$ in (20) is not a simple closed convex curve according to Lemma 1 , as depicted in Figure $1 \mathrm{~b}$.

To further illustrate the proposed result, we set $C=1, d=2000, \omega=3$ in (19) and take different derivative orders of (21). When the derivative order is less than 2 , the curve after the corresponding derivative is still a curve of constant width, as shown in Figure 2a, as well as the blue line in Figure 2b. When the derivative order is equal to 3, the curve is no longer convex, as depicted in the purple line in Figure 2b. Moreover, if the derivative order is increasing, the obtained curve is able to intersect itself, and it is no longer a simple closed curve, as shown in Figure 3a. When the derivative order is continuously increased, the resulting curve is approximated to (24). Namely, the curve is a circle of radius $\left|\frac{C}{2}(1-\omega)(\omega+1)^{n}\right|$ that has turned four times, as shown in Figure $3 b$. In addition, when the derivative order is large enough, the four circles will overlap into one circle.

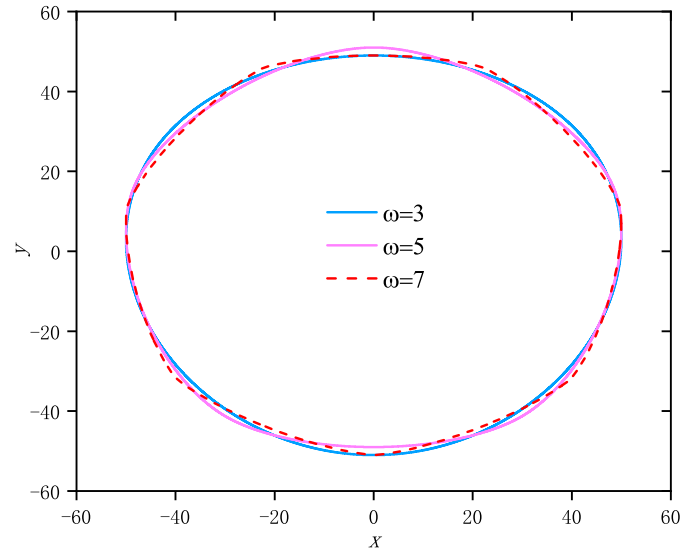

(a) $d=100, c=1, \omega=3, \omega=5$ and $\omega=7$



(b) $d=100, c=1$ and $\omega=9$

Figure 1. Trajectory curves of (20) with different $\omega$.

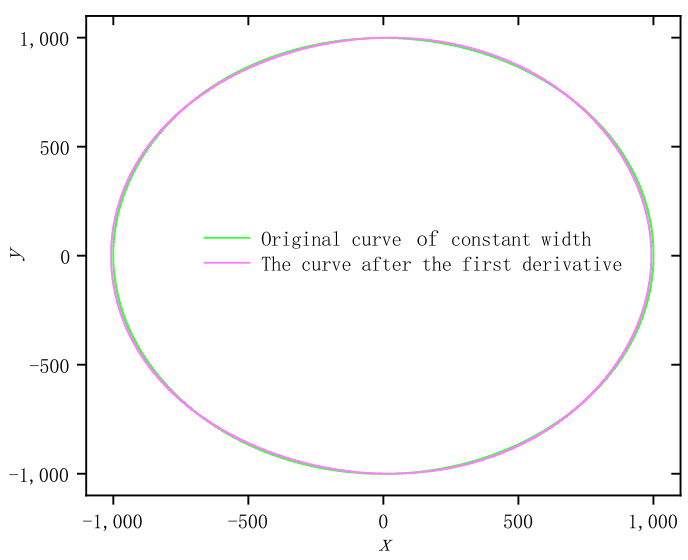

(a) The original curve of constant width and the first derivative curve



(b) The second and third derivative curves

Figure 2. Trajectory curves with different number of derivatives $(d=2000, c=1, \omega=3)$. 


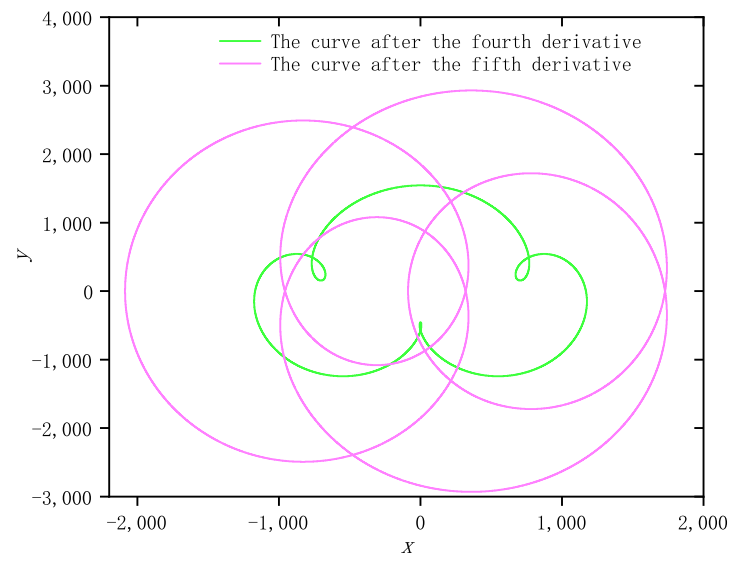

(a) The fourth and fifth derivative curves

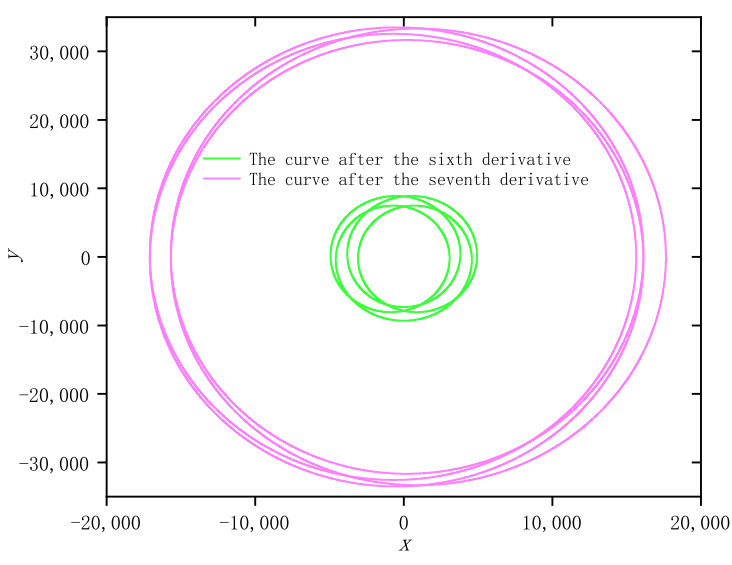

(b) The sixth and seventh derivative curves

Figure 3. Trajectory curves with different number of derivatives $(d=2000, c=1, \omega=3)$.

\section{Conclusions}

This paper innovatively introduces the characteristic equation of a time delay system to analyze the $C^{\infty}$ smooth curve of constant width. We can construct a $C^{r}$ smooth noncircular curve of constant width on the basis of the above analysis. We also prove that there is no $C^{\infty}$ smooth non-circular curve of constant width, which is still a curve of constant width after taking any number of derivatives.

Author Contributions: Conceptualization, T.F. and Y.Z.; methodology, T.F.; software, T.F.; validation, T.F.; formal analysis, Y.Z.; investigation, T.F.; resources, Y.Z.; data curation, T.F.; writing-original draft preparation, T.F.; writing-review and editing, T.F. and Y.Z.; visualization, T.F.; supervision, Y.Z.; project administration, Y.Z.; funding acquisition, Y.Z. All authors have read and agreed to the published version of the manuscript.

Funding: This work was supported by Cultivation Project of Guizhou University ([2019]63), the Science and Technology Program of Guizhou Province ([2018]1047), Research Foundation for Talents of Guizhou University ([2017]61) and the fund project of Key Laboratory of Advanced Manufacturing technology, Ministry of Education, Guizhou University (KY[2018]478).

Institutional Review Board Statement: Not applicable.

Informed Consent Statement: Not applicable.

Data Availability Statement: Not applicable.

Conflicts of Interest: The authors declare that they have no conflict of interest. The funders had no role in the design of the study; in the collection, analysis, or interpretation of data; in the writing of the manuscript, or in the decision to publish the results.

\section{References}

1. Moon, Y.; Kota, S. Automated synthesis of mechanisms using dual-vector algebra. Mech. Mach. Theory 2002, 37, 143-166. [CrossRef]

2. Martini, H.; Mustafaev, Z. A new construction of curves of constant width. Comput. Aided. Geom. Des. 2008, 25, 751-755. [CrossRef]

3. Borovikova, M.; Ibragimov, Z. Convex bodies of constant width and the Apollonian metric. Bull. Publ. Health Soc. 2008, 31, 117-128.

4. Groemer, H.; Wallen, L.J. A measure of asymmetry for domains of constant width. Beitr. Algebra Geom. 2001, 42, 517-521.

5. Rabinowitz, S. A polynomial curve of constant width. Missouri J. Math. Sci. 1997, 9, 3-27. [CrossRef]

6. Paciotti, L. Curves of constant width and their shadows. Whitman Senior Project 2010, 2010, 20.

7. Xu, W.X.; Zhou, J.Z.; Chen, F.W. A kind of constant width "isosceles trapezoid". Sci. China Math. 2011, 41, 855-860.

8. Yang, Y. A Convex Set of Constant Width with an Opposite Top Sector; Wuhan University of Science and Technology: Wuhan, China, 2015. 
9. Mozgawa, W. Mellish theorem for generalized constant width curves. Aequ. Math. 2015, 89, 1095-1105. [CrossRef]

10. Mellish, A.P. Notes on differential geometry. Ann. Math. 1931, 32, 181-190. [CrossRef]

11. Resnikoff, H.L. On curves and surfaces of constant width. arXiv 2015, arXiv:1504.06733. [CrossRef]

12. Kupitz, Y.; Martini, H.; Wegner, B. A linear-time construction of reuleaux polygons. Beitr. Algebra Geom. 1996, $37,415-427$.

13. Pan, S.L. An application of tangential polar coordinates. J. East China Norm. Univ. Nat. Sci. 2003, 1, 13-16.

14. Martini, H.; Montejano, L.; Oliveros, D. Sections of bodies of constant width. In Bodies of Constant Width; Birkhäuser: Cham, Switzerland, 2019. 